\title{
Infrastructureless Pedestrian Navigation to Assess the Response of Alzheimer's Patients to Visual Cues
}

\author{
I.D. McCarthy*, K. Yong ${ }^{\dagger}$, T. Suzuki*, B. Yang*, N. Papafosidos*, D. Boampong*, C. Holloway*, \\ N. Tyler*, A. Carton ${ }^{\dagger}$, D. Voyce ${ }^{\dagger}$, S. Crutch ${ }^{\dagger}$ \\ * Pedestrian Accessibility and Movement Environment Laboratory, Department of Civil, Environmental and Geomatic \\ Engineering, University College London; ${ }^{\dagger}$ Dementia Research Centre, Institute of Neurology, University College London
}

Keywords: Posterior cortical atrophy; Alzheimer's disease; inertial measurement units; way finding; visual cues.

\begin{abstract}
The aim of the study was to understand how patients with dementia were able to navigate in a domestic environment, and whether the presence of visual cues could assist in route finding. A simulated domestic environment was created in the Pedestrian Accessibility and Movement Environment Laboratory (PAMELA) comprising three different types of corridors and an open room with three doors. Three groups of subjects were studied: i) posterior cortical atrophy; ii) typical Alzheimer's disease; iii) age- and gender matched controls. Foot-mounted inertial measurement units (IMUs) were used to measure accelerations in 3D. Data from the sensors was transmitted wirelessly to a laptop computer. Local accelerations were converted to global accelerations, and double integration was performed to calculate displacement; corrections for sensor drift were performed during this process.
\end{abstract}

\section{Introduction}

Alzheimer's disease (AD) is the most common cause of dementia, characterised by the progressive invasion of the cortex by amyloid plaques and neurofibrillary tangles. The most frequent leading symptom of typical Alzheimer's disease (tAD) is memory impairment, consistent with pathological changes within the medial temporal regions. Posterior cortical atrophy (PCA) is a neurodegenerative syndrome most often caused by AD; however, PCA patients show a greater distribution of pathological changes towards the back of the brain in parietal, occipital and occipitotemporal regions. Correspondingly, PCA patients show more prominent deficits in processing visual information compared to $\mathrm{tAD}$, contrasted by relatively preserved memory function [1].

Dementia patients demonstrate pathological changes in components of the ganglia-thalamacortical loop $[2,3]$, resulting in high-level gait disorders. Four clinical features of high-level gait disorders have been proposed by Elble: 1) inappropriate limb movements and interaction with the environment; 2) qualitatively variable performance; 3) hesitation and freezing; 4) inappropriate rescue reactions [4].
Monitoring patient's daily gait would be a great help to evaluate the progress of $\mathrm{AD}$ and the increased risk of injuries from falling. However, traditional methods of gait analysis are impractical for daily monitoring.

Traditional gait analysis, particularly for lower-level gait disorders involving musculoskeletal pathology of the lower limb, is normally performed in a standardized laboratory setting in which participants walk in a straight line at a constant velocity on a flat surface. While this has the advantage of standardization for comparison between laboratories, it does not replicate many daily situations that may pose unique problems for individuals with cognitive deficits. Lighting levels, the presence or absence of shadows, other distractors, and requirements for decision-making are important factors in determining navigational ability in tAD, possibly due to impaired executive function, memory and visual processing $[5,6]$. Perceptual factors of the environment may carry particular significance in PCA, with patients exhibiting deficits in basic visual, visuo-spatial and visuoperceptual function [1]. Experimental settings investigating such factors are difficult to set up in a standard gait laboratory, and other techniques are required to assess gait.

In Parkinson's disease, most gait studies have investigated spatio-temporal parameters of gait from measurements of people walking on a pressure mat. Typically, the effect of dual-tasking on gait is measured, and the effects of medication can be assessed. Although such systems are portable, and so may be set up in, for example, a care home, there are still constraints with regard to flexibility of situations where they can be used [7].

Sensors attached directly to the participants would, in principle, enable assessment of spatio-temporal gait characteristics under any environment. Inertial measurement units (IMUs) have been used in gait studies for the measurement of joint angles in a study of healthy ageing [8]. Such devices are very portable, and have been used previously in a day centre for the elderly. No infrastructure was required in the building; data was recorded by a laptop, but in theory data could collected remotely with an appropriate high-speed internet connection.

Use of IMUs in pedestrian navigation has also been described [9]. This application was designed for use by firstresponders in emergency situations. It was described as an "infrastructureless" technique, as the devices could be attached directly to the emergency worker to monitor their position in, for example, a burning building, and would 
require no pre-existing or functional position detection system within the building. It therefore seems feasible that IMUs could be used both to monitor the position of Alzheimer's patients as well as characterise their gait in specific situations.

The long-term aim of the work is to develop strategies that would create a dementia friendly environment, in order to enable patients to function more independently in a home environment. In this paper, preliminary experiments are described, in which IMUs were used to evaluate how Alzheimer's patients responded to visual cues designed to aid their navigation.

\section{Methodology}

\subsection{PAMELA platform.}

In order to provide a safe and controllable environment for the assessment of dementia subjects, two simulated domestic environments were set up in the Pedestrian Accessibility and Movement Environment Laboratory (PAMELA) at University College London. The main platform of PAMELA consisted of 54 configurable modules $(9 \times 6)$ that are $1200 \mathrm{~mm}$ $\times 1200 \mathrm{~mm}$ squares. Wood boards of $55 \mathrm{~mm}$ in thickness were put on top of the concrete modules and then floored with dark-blue domestic carpet. The entire area of floor was gapfree and levelled.

The platform was arranged in two configurations for two experimental tasks. For Task 1, the platform was configured to provide corridors with $2 \mathrm{~m}$ high walls using free-standing oak-coloured wooden panels $(2000 \mathrm{~mm}$ height, $1200 \mathrm{~mm}$ width, $42 \mathrm{~mm}$ thickness) as walls. Walls were of high contrast to the floor carpet, consistent with recommendations for the design of residential environments for individuals with sight loss. This task was comprised of three corridors: a $6 \mathrm{~m}$ straight corridor (ST), a 9m U-shaped corridor with two $90^{\circ}$ turns in the same direction (U), and a 9m "dog-leg" corridor with two $90^{\circ}$ turns in opposite directions (S). These are illustrated schematically in Figure 1. The task was conducted under two levels of average horizontal illuminance (15 or $150 \mathrm{lux}$ ) and visual cue conditions (cue vs. no cue). The visual cue consisted of floor-level LED lights displaying a dynamic light pattern that continuously moved towards the end of each corridor.

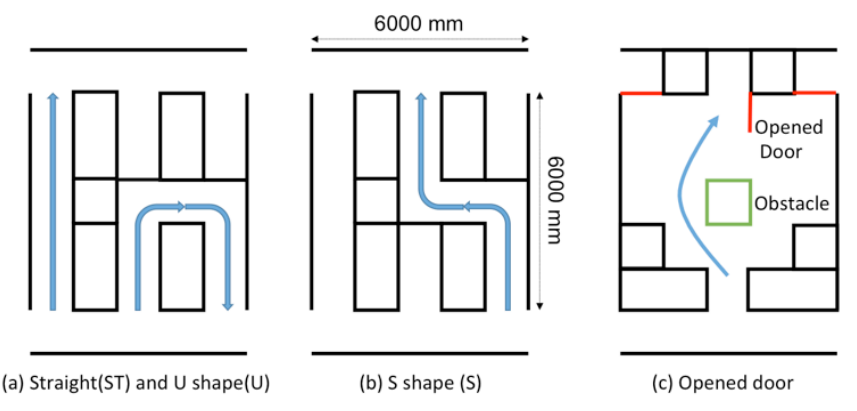

Figure 1. Schematic arrangement of the PAMELA platform for corridor tasks.
For Task 2, an open room was created with three doors along one wall, one of which would be open; this is also illustrated in Figure 1. Participants were positioned on the opposite side of the room to the doors, and were told to walk towards the open door. The task was conducted under three visual cue conditions (contrast cue, motion cue, no cue) and two obstacle conditions (obstacle vs. no obstacle). Cues were presented on the handle of the door, while obstacles were placed between the participant and the open door.

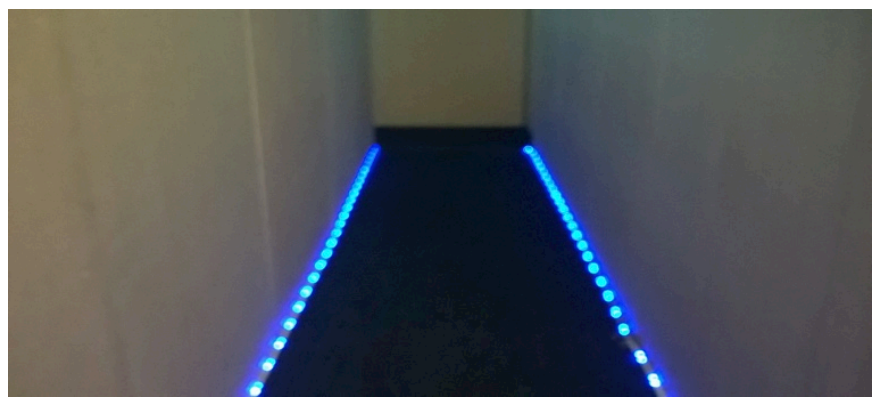

Figure 2. LED lights positioned at floor level of a corridor in order to assess the effect of this visual cue on participant performance.

\subsection{Protocol for dementia patients}

Two groups of dementia patients were studied: 11 with posterior cortical atrophy (PCA), and 10 with typical Alzheimer's disease (tAD). These were compared with an age- and gender-matched control group. For Task 1, participants were asked to 'walk towards the end of the corridor' after the experimenter counted down 'three, two, one' and then indicated the beginning of trial by saying 'start'. The end of each trial was when participants crossed the finishing line of the route. Participants walked down both directions of each corridor. For Task 2, participants (8 PCA, 10 tAD) were asked to walk towards the open door. The end of each trial was when participants reached the closest part of the door frame.

\subsection{Assessment of Movement}

Movement of the participants was measured by attaching inertial measurement units (IMUs) to each heel, as illustrated in Figure 3. The IMUs comprise tri-axial accelerometers, gyroscopes and magnetometers. Data was communicated wirelessly to a base station connected to a laptop computer. Data was then exported in .txt format for analysis by customwritten MATLAB scripts. 3D accelerations in local IMU coordinates were converted to global coordinates by applying a rotation matrix. Accelerations were then integrated to 


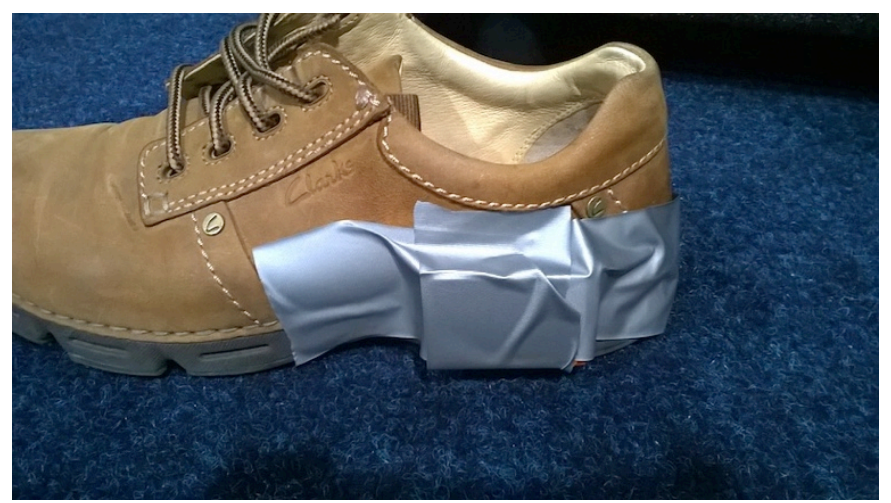

Figure 3. Attachment of the IMU to the heel of the shoe. An alignment jig was placed under the IMU during attachment to keep it horizontal.

calculate velocity. Inaccuracies in sensor alignment [10] resulted in drift in calculated velocities, as illustrated in Figure 4.

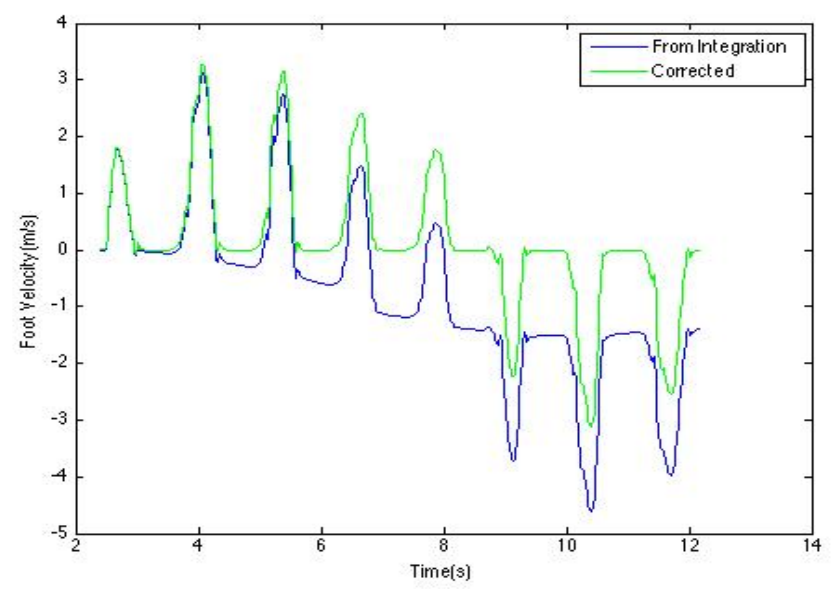

Figure 4. Example of sensor drift in the calculation of foot velocity. Stationary periods for the foot were identified from a defined threshold of resultant acceleration values, and these stationary periods were then used to correct the velocity data.

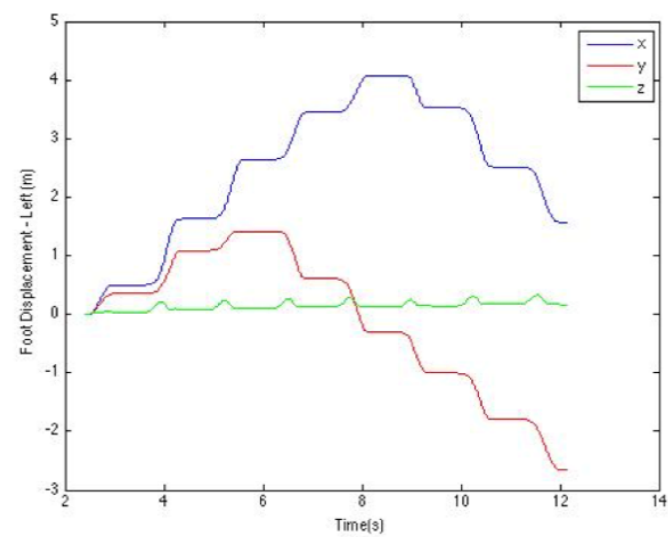

Figure 5. Foot position during walking in $3 \mathrm{D}$ calculated from IMU data; $\mathrm{x}$ and $\mathrm{y}$ are the horizontal axes, and $\mathrm{z}$ the vertical axis.
Periods of zero velocity, when the foot was in contact with the ground, were estimated, and velocity corrected (Figure 4). Corrected velocities were integrated again, to obtain foot position in $3 \mathrm{D}$, as illustrated in Figure 5.

The position of the feet on the platform could then be estimated from the $\mathrm{x}$ and $\mathrm{y}$ data (Figure 6). Clusters of points identify the stationary phases of the foot, indicating how spatio-temporal gait parameters may be related to position on the platform. It should also be noted that in addition to plotting the position in the horizontal plane, foot height can be obtained from the movement in the $\mathrm{z}$ direction.

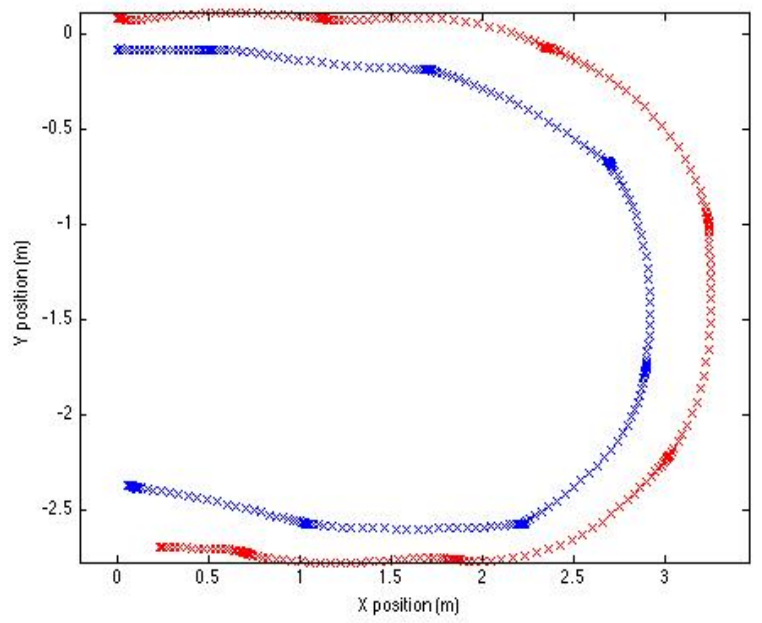

Figure 6. Example of path taken by a control subject when walking along the U-shaped corridor, showing both left and right feet.

\section{Results}

In general and under baseline conditions, PCA and tAD patients walked slower than controls, with shorter step lengths and longer step times. Variation in step length and time during walking was also greater for patients. Control subjects tended to maintain step time when walking on the $U$ and $S$ corridors, whereas patients increased step time; all groups showed a reduction of step length when walking along the $U$ and $\mathrm{S}$ corridors.

When considering how participants navigated on more complex tasks such as $\mathrm{U}$ and $\mathrm{S}$ corridors, and the doors tasks, a number of patients performed in different ways qualitatively. All controls followed a smooth arc when walking around the corners in the $\mathrm{U}$ and $\mathrm{S}$ corridors, but a number of patients in both groups followed the walls closely, making sharp right-angle turns at the corners. This may reflect patients becoming disorientated at turns, and/or having difficulty perceiving turns. When considering the response to visual cues, it is informative to consider a couple of case studies:

i) This PCA patient was particularly slow and hesitant, even when he was walking along a simple straight corridor. Two examples of the path taken by him walking along the $\mathrm{S}$ 
corridor in Task 1 are shown in Figure 7. In this instance, the provision of the cue was counter-productive; he became disorientated at the initial turning, repeatedly fixating the dynamic light pattern.

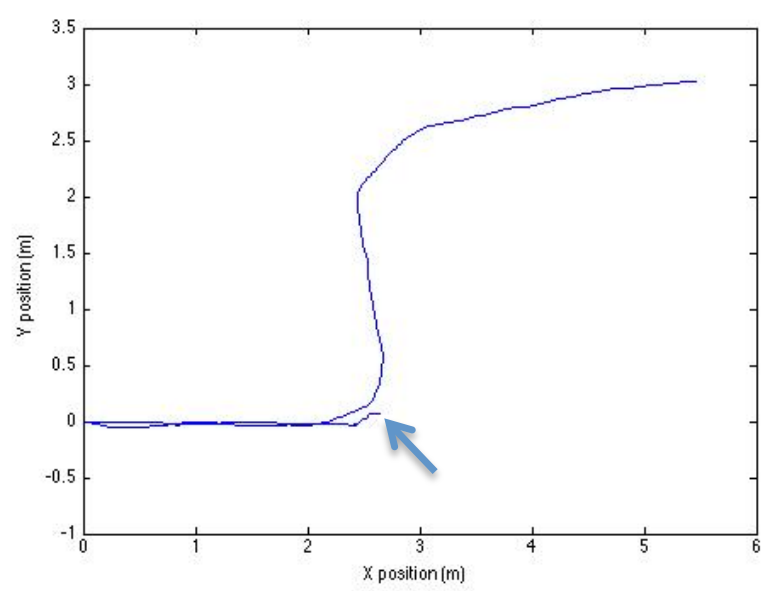

Figure 7. Example paths taken by a patient walking the $\mathrm{S}$ corridor, one in which the LED floor lighting cue was switched on, and the other when it was switched off. The arrow indicates the final position of the participant when the cue was on.

ii) Another patient showed clear deficits in navigational ability. Two paths of trials from Task 2 are shown in Figure 8. One of the trials was successfully completed, but in the other the patient was not able to reach the open door within 60 seconds. Task performance was slow, even in the successful trial; there is a point where the patient stood still for a number of seconds before resuming the path towards the door. Analysis of Task 2 preliminary data suggests that the presence of a cue results in an overall improvement in patients' task performance.

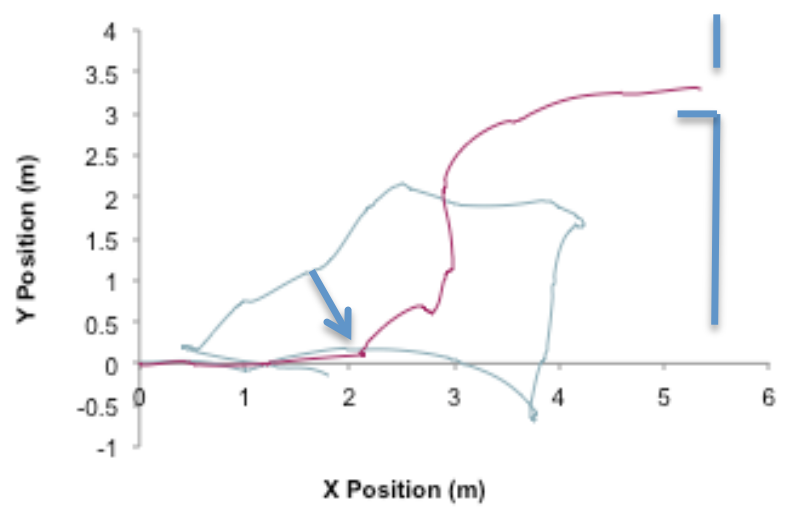

Figure 8. Examples of attempts to walk to an open door by one patient. The arrow indicates a point in the successful trial where the patient stopped for several seconds.

\section{Discussion and Conclusion}

In this study we have used IMUs to assess function of dementia patients when walking in a controlled environment, and successfully demonstrated the functions of gait monitoring for tAD and PCA patients. Foot-mounted sensors enabled both the measurement of spatio-temporal parameters of gait, and the tracking of position of participants within the environment. This enables gait parameters to be related to particular features within that environment.

Patients' symptoms can vary dynamically from day to day, and very small dynamic abnormalities can occur suddenly. With the IMU measurements, continuous and accumulated gait monitoring may have the potential to detect and track signs of disease in TAD and PCA, and evaluate daily activities and autonomy.

A possible limitation of this study is the accuracy in IMU measurement. The extracted parameters in healthy controls were used as a baseline for comparison to avoid misinterpretation of results. The absolute accuracy will need to be evaluated further in the future.

The sensors were small, and could be attached to a shoe, or even potentially fitted within a shoe. Data was transmitted wirelessly to a base station. It is therefore a very portable system, and therefore position-tracking could be set up anywhere, not requiring specific infrastructure in the building for this to happen. Although we have performed these studies in a controlled environment, it would be feasible to perform such assessments at home or in a residential care setting, and there are possible applications to develop automatic dynamical clinical assessments by machine learning with accumulated data. Indeed, this might be particularly advantageous when evaluating future interventions to support the independence of individuals with a diagnosis of dementia.

\section{Acknowledgements}

The study was funded by an ESRC/NIHR grant (ES/L001810/1), Alzheimer's Research UK Senior Research Fellowship and ESRC/NIHR grant (ES/K006711/1) to SC, and UCLH/UCL Department of Health's NIHR Biomedical Research Centres funding scheme. The Dementia Research Centre is an Alzheimer's Research UK Coordinating Centre. The Dementia Research Centre is supported by Alzheimer's Research UK, Brain Research Trust, and The Wolfson Foundation. This work was supported by the NIHR Queen Square Dementia Biomedical Research Unit.

\section{References}

[1] Crutch, S., Lehman, M., Schott, J.M., Rabinovici, G.D., Rossor, M.N., and Fox, N.C. 'Posterior cortical atrophy', Lancet Neurol, 2012, 11, pp. 170-178.

[2] Meyer, J.S., and Barron, D.W. 'Apraxia of gait: a clinicopathological study’, Brain, 1960, 83, pp 261-284. 
[3] Nutt, J.G., Marsden, C.D., and Thompson, P.D. 'Human walking and higher level gait disorders, particularly in the elderly', Neurology, 1993, 43, pp 268-279.

[4] Elble, R.J. Gait and dementia: moving beyond the notion of gait apraxia. J Neural Transm, 2007, 114, pp1253-1258.

[5] Pai, M.C., and Jacobs, W.J. 'Topographical disorientation in community-residing patients with Alzheimer's disease', Int J Geriatr Psychiatry, 2004, 19, pp 250-5.

[6] Passini, R., Rainville, C., Marchand, N., and Joanette, Y. 'Wayfinding in dementia of the Alzheimer type: planning abilities'. J Clin Exp Neuropsychol, 1995, 17, pp. 820-832.

[7] Chien, S.-L., Lin, S.-Z., Liang, C.-C., Soong, Y.-S., Lin, S.-H., Hsin, Y.-L. et al. 'The efficacy of quantitative gait analysis by the GAITRite system in evaluation of parkinsonian bradykinesia' Parkinsonism and Related Disorders, 2006, 12, pp. 438-442.

[8] Beauregard, S. 'Infrastructureless Pedestrian Positioning', PhD Thesis, University of Bremen, 2009.

[9] Monda, M., Goldberg, A., Smitham, P., Thornton, M., and McCarthy, I. 'Use of inertial measurement units to assess agerelated changes in gait kinematics in an active population', $\mathrm{J}$ Ageing Phys Act, 2015, 23, pp 18-23.

[10] Woodman, O.J. 'An Introduction to Inertial Navigation', University of Cambridge Computer Department, Technical Report 696, ISSN 1476-2986. 